\title{
The effects of glass powder on some mechanical properties of engineering thermoplastics
}

\author{
L.Karunanayake \\ Department of Chemistry, University of Sri Jayewardenepura, Gangodawila, Nugegoda.
}

Revised: 13 March 2006 ; Accepted: 20 December 2006

\begin{abstract}
The manufacture of composite materials based on plastics using fillers such as glass fibre, carbon black and wood dust is done to impart better properties or/and to reduce the cost of material. In this study, glass powder was used as a filler in four engineering plastics, namely, Nylon 6, Nylon 66, Polybutylene terephthalate and Polyarylamide. Mixing of glass powder and polymers was achieved by melt blending using a twin-screw extruder. Properties such as tensile properties, flexural properties, mould shrinkage, thermal expansion and specific gravity were ascertained using injection-moulded samples. It was found that glass powder can be mixed effectively with the above four plastics. The desirable effects of mixing of glass powder with the four thermoplastics was evident by the significant improvement of some properties. The composites of polyarylamide showed more desirable properties indicating high compatibility.
\end{abstract}

Keywords: Blending, engineering plastics, glass powder, physical properties, polymer composites.

\section{INTRODUCTION}

The incorporation of glass fibre in polymeric materials to enhance properties of end products has been well-studied ${ }^{1-7}$. Some of the polymer glass fibre composites that have been studied contain a wide range of polymers such as Nylon 6 (PA6) ${ }^{1-6}$, Polybutylene terephthalate (PBT) ${ }^{1}$, Polypropylene ${ }^{3}$ and Epoxy resins ${ }^{7}$. Glass fibre reinforced polymer nanocomposites have also been recorded. However, the use of glass powders in polymer composites is less common.

Fillers such as glass fibre improves properties of thermoplastics like falling weight impact strength $^{5}$, compressive strength ${ }^{8}$, albeit with some adverse effects, for example increase in density, decrease in melt flow and increase in brittleness ${ }^{6}$. Further, the incorporation of glass fibres to engineering plastics is found to be essential in semi-structural applications to improve strength, stiffness and fatigue resistance ${ }^{1}$. Scientists ${ }^{1}$ have reported that when glass fibres are used as fillers, some properties of the material depend significantly on the orientation of fibres, which in turn depends on, flow direction, geometrical feature of the mould and positions of gates. Although, the above factors can affect the distribution of glass powders that are used in this study, the problem of orientation of the glass fibre becomes a trivial factor. Further, very fine powder like glass beads have been used in applications such as dental fillings ${ }^{8}$ and prosthetic fixation or as a bone substitute in orthopaedics, ${ }^{9}$ where the penetration of materials to very fine cavities and the strengthening of the polymeric material are important. Glass powder has significant advantages over glass fibres. In some studies, the suggested glass powder level is as high as $70 \%(\mathrm{w} / \mathrm{w})$ and this has been achieved by activating the surface of glass powders using silane-coupling agents 9 However, these biological applications do not need the type of high physical properties that applications of engineering plastics demand.

The objective of this investigation was to study the effects of incorporation of glass powders on the properties of four engineering thermoplastics at four different loading levels. The properties of four thermoplastic composites with different glass powder levels were measured. Since glass powder is a material that can be produced from waste or scrap glass, it can be treated as an inexpensive material that can be used with different plastics.

\section{METHODS AND MATERIALS}

The glass powder used in this study was of $50 \mu \mathrm{m}$ average particle size and 2.5 specific gravity. 
Table 1: Temperature settings for the injection moulding machinc

\begin{tabular}{|c|c|c|c|c|c|c|}
\hline Material & Barrel Rear ${ }^{\circ} \mathrm{C}$ & Barrel ${ }^{\circ} \mathrm{C}$ & Barrel Middle $/{ }^{\circ} \mathrm{C}$ & Barrel Front/ ${ }^{\circ} \mathrm{C}$ & Nozzle $/{ }^{\circ} \mathrm{C}$ & Mould $/{ }^{\circ} \mathrm{C}$ \\
\hline PA6 & 270 & 275 & 280 & 270 & 260 & 60 \\
\hline PA 66 & 280 & 285 & 290 & 280 & 270 & 60 \\
\hline PBT & 210 & 220 & 225 & 210 & 200 & 60 \\
\hline PAA & 240 & 245 & 250 & 250 & 250 & 90 \\
\hline
\end{tabular}

Four engineering plastics Nylon 6 (PA 6), Nylon 66 (PA 66), Polybutylene terephthalate (PBT) and Polyarylamide (PAA) were blended with different amounts

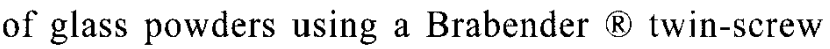
extruder. The barrel temperatures used for the extruder are similar to the barrel temperatures of the injectionmoulding machine (see Table 1). The feeding speed was kept at $50 \mathrm{rpm}$. Samples containing 15\%,30\% and 50\% (w/w) glass powder were prepared for each polymer by mixing them in a polyethylene bag. The total weight of the sample was kept at $2 \mathrm{~kg}$. A $2 \mathrm{~kg}$ sample of each virgin polymer was taken as the control. Before charging the extruder for melt blending, all samples were dried overnight at $100{ }^{\circ} \mathrm{C}$ in an air-circulating oven. Feeding the hopper was done very carefully to minimize bridge formation in the metering area. Special attention was required during the extrusion of highly loaded polymers (i.e. $50 \%$ ).

The extruded polymer strands were cooled using a water bath and subsequently granulated using a granulator. The granules obtained were dried for $12 \mathrm{~h}$ in an air circulating oven operating at $100^{\circ} \mathrm{C}$ to remove the absorbed water. Test specimens were injection moulded to determine flexural properties ${ }^{10}$, Izod impact strength ${ }^{11}$, tensile properties ${ }^{12}$, density ${ }^{13}$, coefficient of linear thermal expansion ${ }^{14}$ and moulding shrinkage ${ }^{15}$. The injection moulding machine was programmed to obtain temperature settings given in Table 1. Dimensions of test specimens prepared were in accordance with corresponding ISO standards. All the above tests were done according to the ISO standards.

\section{RESULTS}

The results of the properties measured are presented as a ratio to facilitate the following.

1. Comparison of effects of glass powder content on the properties of individual polymers.

2. Comparison of effects of glass powder filling on the properties of four polymers.
The ratio is calculated using the following equation.

Property ratio $=\quad$ Value of the actual Property

Value of the Property for the virgin polymer

\section{DISCUSSION}

The separation of high-density glass powder from the low-density plastic pellets was encountered during the feeding of the materials for melt mixing in the extruder. This was very significant with higher filler loadings and special attention was given to ensure that the feed to the hopper of the extruder was as homogeneous as possible. Further, it was observed that the formation of bridges by the fine glass powder blocks the metering area when the homogeneity of mixture was poor. This results in very short strands of the polymer making continuous process of granulation impossible.

According to Figure 1, it is clear that the mould shrinkage decreases with the increase of glass powder content. The highest decrease was observed for the PAA and the lowest decrease was recorded for PA66. This shows that the incorporation of glass powder in engineering plastics can significantly reduce the mould shrinkage.

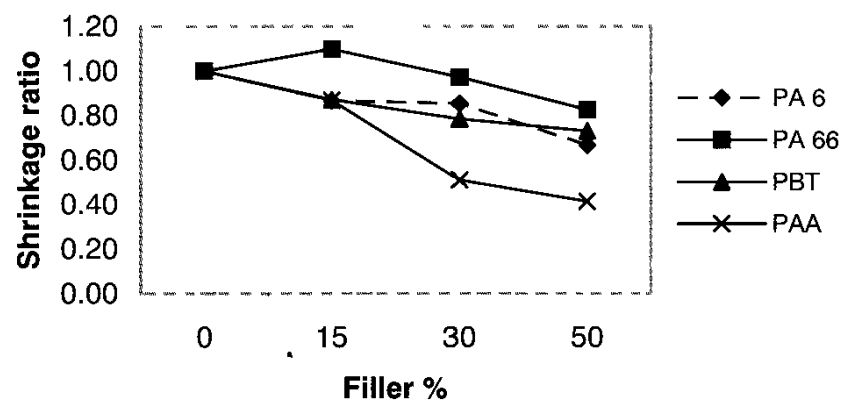

Figure 1: Effect of filler content on mould shrinkage 
In Figure 2, although the polymer PAA shows a remarkable increase in Izod notched impact strength with the increase of glass powder content, the other three polymers show steady decrease showing very similar patterns. The latter is to be expected as the brittleness of the filled samples are found to increase with the filler content and the resistance to crack propagation decreased ${ }^{4.6}$, although the former is unexpected. That can be due to the fact that the incorporation of glass filler to PAA has imparted a resistance to crack propagation ${ }^{6}$.

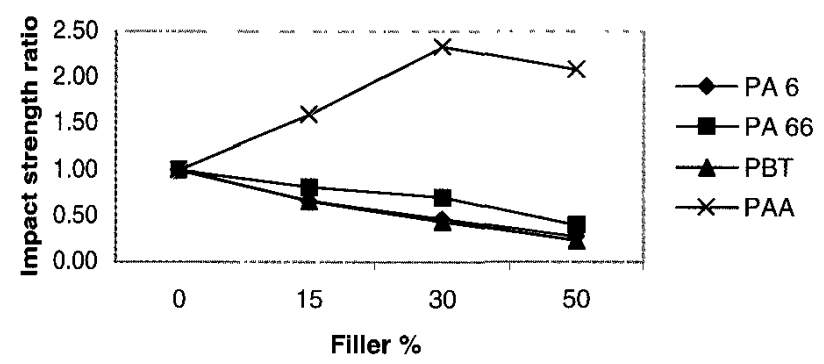

Figure 2: Effect of filler content on impact strength (Izod-notched)

Figure 3 shows a much larger variation of Izod unnotched impact strength values, and confirms the pattern shown by PAA and the other three polymers seen in Figure 2. The increase in impact strength values of PAA with the increase of filler content can be attributed to increase in interfacial adhesion between the polymer and the glass filler ${ }^{3}$. It shows that the glass powder is more compatible with PAA than the other three polymers. On the other hand, the decrease in unnotched Izod impact strength is normal for polymers with less compatibility with glass powder as the fracture toughness is reduced due to the incorporation of glass powder ${ }^{6}$.

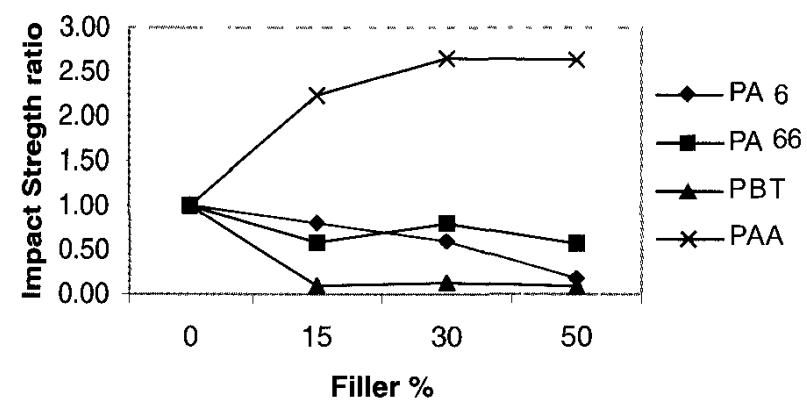

Figure 3: Effect of filler content on impact strength (Izod-unnotched)

According to the tensile values illustrated in Figure 4, although there is no linearity of increase, the incorporation of the filler has increased the tensile values remarkably for composites of PAA. The tensile strength values of the other three polymers have decreased with the incorporation of glass powder compared to that of their

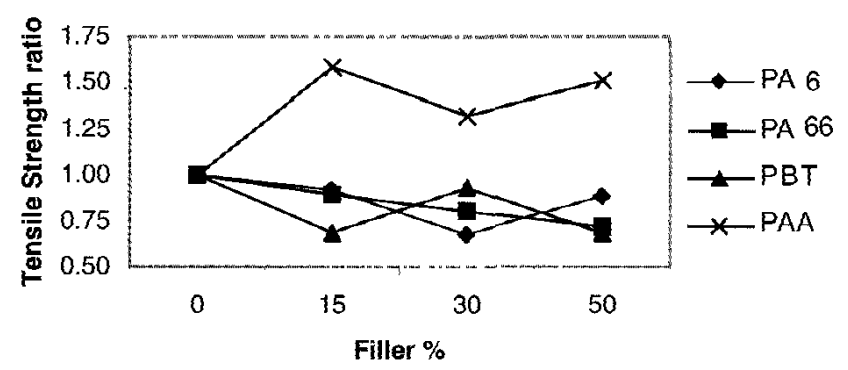

Figure 4: Effect of filler content on tensile strength

virgin samples although there is no linearity in the decrease, as well. These results also confirm the findings of impact strength experiments. Scientists ${ }^{3}$ have attributed the increase of mechanical properties of their blends of glass fibre reinforced nylon $6 /$ propylene blends to enhancement of stress transfer. Therefore, the enhancement of mechanical properties such as impact strength and tensile strength in glass powder enriched PAA samples can be due to strong interaction and adhesion between the two materials.

As depicted in the Figure 5, the elongations at break values recorded are lower than those of corresponding virgin polymers, except in the case of $15 \%(w / w)$ filled PAA sample. However, less reduction of elongation at break values of PAA samples is noticeable. This clearly supports the above argument. A previous study ${ }^{6}$ has attributed the reduction of toughness to the presence of rigid particles in the composites. Moreover, the reduction of toughness is the cause for the reduction of ultimate elongation.

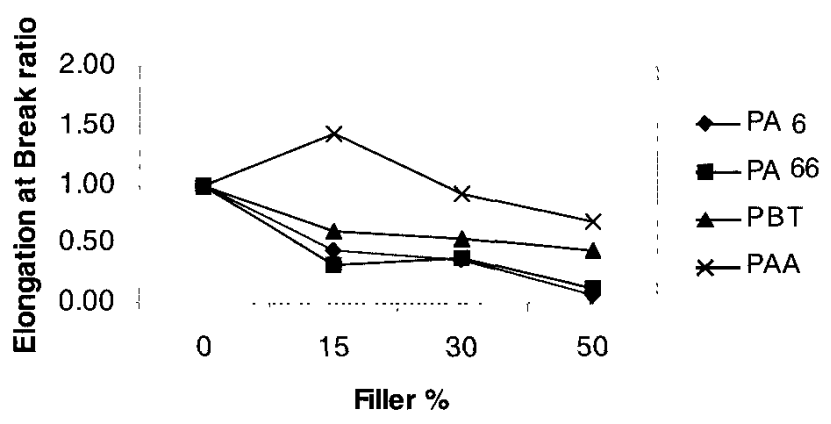

Figure 5: Effect of filler content on elongation at break

As in the properties discussed earlier, flexural strength values of PAA sample illustrated in the Figure 6 have shown a clear increase. However, in contrast to the earlier results, PA6 has also shown an increase of values with increase of filler content after an initial set back. PA66 has shown decrease with filler content while values for PBT have shown an irregular pattern. This is in accordance with the argument of scientists ${ }^{\prime}$ where they have claimed that flexural and tensile results can show 
significant differences due to the method of measuring the flexural strength.

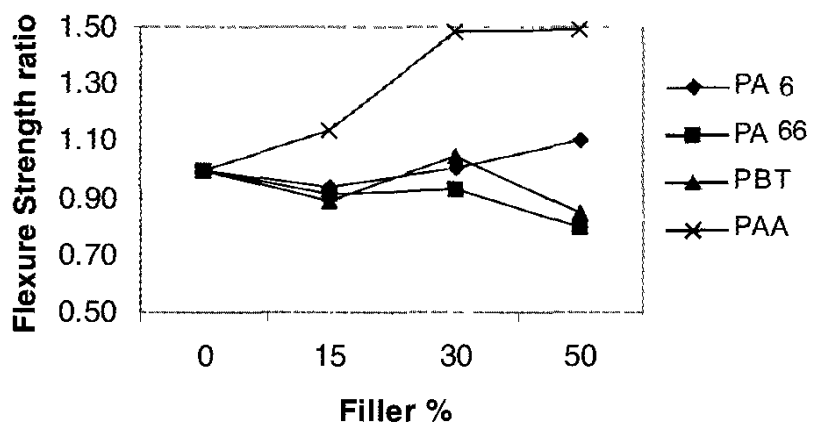

Figure 6: Effect of filler content on flexural strength

Figure 7 illustrates that the samples of PBT and PA6 show a steady increase in flexural modulus values with the increase of glass powder content where as the samples of PAA and PA66 show a lesser increase. As the decrease in flexural modulus is anticipated with the increase of impact strengths ${ }^{5}$, the decrease of flexural modulus of PAA with the increase of filler content is desirable. However, it is remarkable that this decrease is very similar to that of PA66, which shows much lower impact strength values with the filler loadings.

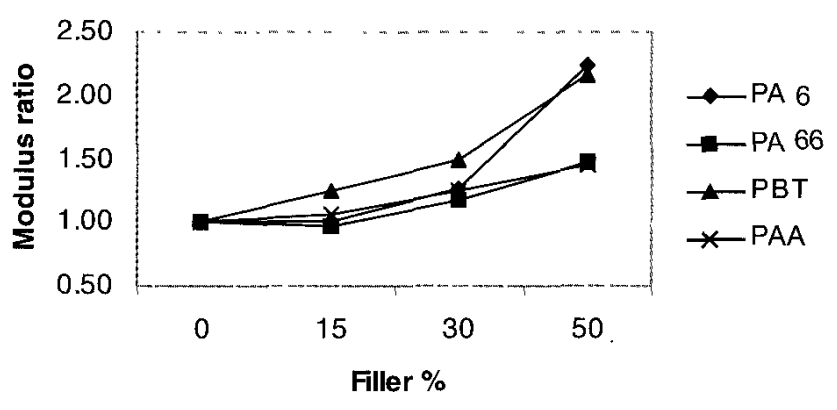

Figure 7: Effect of filler content on flexural modulus

Composites of PBT, PA6 and PAA have shown considerably low values of thermal expansion coefficient compared to their virgin materials (Figure 8). These values reduce with the increase of glass powder content incorporated. The lowest values are found to be for the samples made with PAA.

The curves, which are very close to each other, that illustrate increases of specific gravity values with glass filler content seen in Figure 9 provide the evidence of homogeneity and effectiveness of mixing glass powder to plastics. However, the increase of specific gravity of plastics has been treated as a significant drawback in incorporation of glass fillers to plastics ${ }^{6}$.

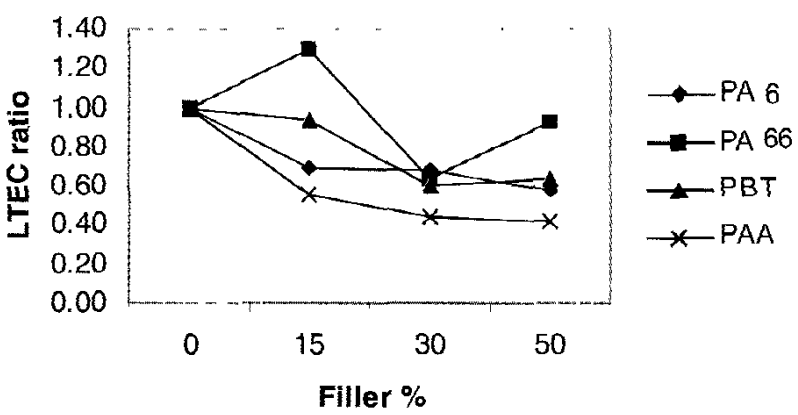

Figure 8: Effect of filler content on linear thermal expansion coefficient (LTEC)

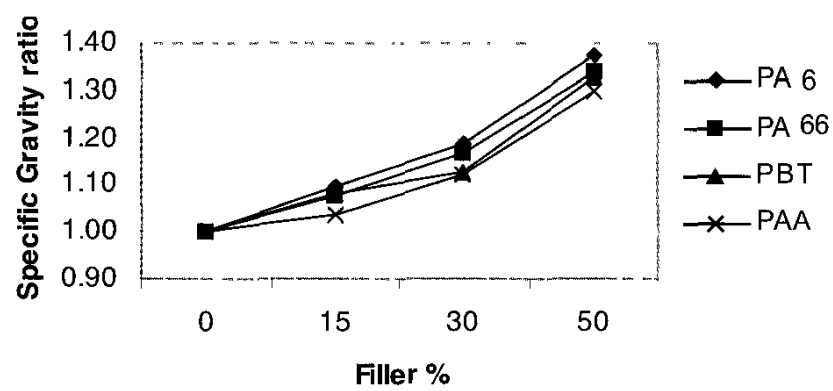

Figure 9: Effect of filler content on specific gravity

\section{CONCLUSION}

The significant property changes that occurrs, in particular the changes of specific gravity, due to the incorporation of glass powder have provided evidence for successful mixing of glass powder to PA6, PA66, PBT and PAA. Further, it was found that the polymer PAA is highly compatible with glass powder. This compatibility has imparted improvements in properties such as mould shrinkage, impact strength, tensile properties and thermal expansion. All four polymers have shown improvement in mould shrinkage, albeit they have shown increase in density, which is a disadvantage, when mixed with glass powder.

\section{Acknowledgement}

The School of Polymer, University of North London, London, where the author carried out initial work of this project is acknowledged.

\section{References}

1. Wyzgoski M.G., Krohn J.A. \& Novak G.E. (2004). Fatigue of fibre-reinforced injection moulded plastics. I: stresslifetime data. Polymer Composites 25(5): 489-498.

2. Pegoretti A., Fambri L. \& Migliaresi C. (2000). Interfacial stress transfer in nylon-6/e-glass microcomposites: effect of temperature and strain rate. Polymer Composites 21(3): $466-475$ 
3 Abbacha N \& Fellahı S (2002) Synthesis of PP-g-MAH and evaluation of its effect on the properties of glass fibre reinforced nylon $6 /$ propylene blends Macromolecula Symposia 178 131-138

4 Gabbert JD, Gamer A Y \& Hednck R M (1983) Remforced nylon 6 block copolymers Polvmer Composites 4(3) 196199

5 Mascia L \& Kinsella M (1993) Thermoplastrcs composites with ductile fibre coatings based on UHMWPE A study on short fibre GR nylon 6 Polymer Composites 14(2) 94-100

6 Akkapeddı M K (2000) Glass fibre reınforced polymer nanocomposites Polymer Composites 21(4) 576585

7 Moehlenpah A E, Ishai O \& Dibenedetto A T (1971) The effect of time and temperature on the mechanical behavior of epoxy composites Polymer Engineering and Science 11(2) 129-138

8 Akunmade A O, Braybrook J H \& Nicholson J W (1994) Glass polyalkenoate dental cements based on physical blends of poly(acrylic acıd) and poly(vınyl phosphonıc acid) Polymer International 34(1) 8188
9 Shnzato S, Nakamura T, Kokubo T \& Kitamura Y (2002) PMMA based broactive cement Etfect of glass bead filler content and histological change with time Journal of Biomedical Materials Rescarch $59225-232$

10 International Organization for Standardization (2001) Plastics - Determination of exural properties ISO 178

11 International Organzation for Standardization (2000) Plastus - Determination of Izod impact strength ISO 180

12 International Organization for Standardization (1993) Plastics - Determination of tensile properties ISO 527

13 International Organization for Standardization (2004) Plastics - Method for determining the density of non-cellular plastics ISO 1183

14 International Organization for Standardization (1999) Plastıcs - Thermomechanical analysis (TMA) Part 2 Determination of coefficient of linear thermal expansion and glass transition temperature ISO 113592

15 International Organization for Standardization (2001) Plastics Injection moulding of test specimens of thermoplastic materials Part 4 Determination of moulding shrinkage ISO 2944 